OPEN ACCESS

Edited by:

Tao Xu,

Anhui Medical University, China

Reviewed by:

Jinlong Li,

Southern Medical University, China

Carlo Gabriele Tocchetti,

University of Naples Federico II, Italy

${ }^{*}$ Correspondence: JiBiao Wu

wujibiao1963@163.com

Zhen Zhang

zhangzhen19801981@126.com

Specialty section:

This article was submitted to Inflammation Pharmacology,

a section of the journal

Frontiers in Pharmacology

Received: 28 June 2021

Accepted: 19 August 2021

Published: 01 September 2021

Citation:

Liu J, Chen Z, Li Y, Zhao W, Wu J and Zhang $Z$ (2021) PD-1/PD-L1

Checkpoint Inhibitors in

Tumor Immunotherapy.

Front. Pharmacol. 12:731798.

doi: 10.3389/fphar.2021.731798

\section{PD-1/PD-L1 Checkpoint Inhibitors in Tumor Immunotherapy}

\author{
Jinhua Liu ${ }^{1,2}$, Zichao Chen ${ }^{1,3}$, Yaqun $\mathrm{Li}^{1,2}$, Wenjie Zhao ${ }^{1}$, JiBiao Wu ${ }^{1 *}$ and Zhen Zhang ${ }^{1 *}$ \\ ${ }^{1}$ Innovation Research Institute of Traditional Chinese Medicine, Shandong University of Traditional Chinese Medicine, Jinan, \\ China, ${ }^{2}$ College of Pharmacy, Shandong University of Traditional Chinese Medicine, Jinan, China, ${ }^{3}$ Experimental Center, \\ Shandong University of Traditional Chinese Medicine, Jinan, China
}

Programmed death protein 1 (PD1) is a common immunosuppressive member on the surface of T cells and plays an imperative part in downregulating the immune system and advancing self-tolerance. Its ligand programmed cell death ligand 1 (PDL1) is overexpressed on the surface of malignant tumor cells, where it binds to PD1, inhibits the proliferation of PD1-positive cells, and participates in the immune evasion of tumors leading to treatment failure. The PD1/PDL1-based pathway is of great value in immunotherapy of cancer and has become an important immune checkpoint in recent years, so understanding the mechanism of PD1/PDL1 action is of great significance for combined immunotherapy and patient prognosis. The inhibitors of PD1/PDL1 have shown clinical efficacy in many tumors, for example, blockade of PD1 or PDL1 with specific antibodies enhances $T$ cell responses and mediates antitumor activity. However, some patients are prone to develop drug resistance, resulting in poor treatment outcomes, which is rooted in the insensitivity of patients to targeted inhibitors. In this paper, we reviewed the mechanism and application of PD1/PDL1 checkpoint inhibitors in tumor immunotherapy. We hope that in the future, promising combination therapy regimens can be developed to allow immunotherapeutic tools to play an important role in tumor treatment. We also discuss the safety issues of immunotherapy and further reflect on the effectiveness of the treatment and the side effects it brings.

Keywords: PD-1 inhibitor, PD-L1 inhibitor, biomarkers, immune checkpoint, tumor immune escape, immune-related toxicity, immunotherapy

\section{INTRODUCTION}

Through the process of tumor immune editing, tumor cells acquire multiple methods of evading host immunity in the tumor microenvironment (TME) (Dunn et al., 2002). Studies on tumor immune escape have shown that PD1/PDL1-mediated immune checkpoint in TME is an important component of the tumor immune escape mechanism (Inaguma et al., 2018; Prestipino and Zeiser, 2019; Zhang et al., 2020). Early preclinical evidence suggests that activation of PD1/PDL1 signaling pathway may be the mechanism by which tumors escape the antigen-specific T cell immune response (Dong et al., 2002; Iwai et al., 2002). PD1 on immune cells interacts with PDL1 on tumor cells can protect tumor cells from killing by immune cells (Carlomagno et al., 2017; Wang et al., 2018a; Takeuchi et al., 2020). PD1 was first described in the early 1990s as it is expressed in the course of inducing apoptosis in T cell hybridoma (Ishida et al., 1992; Agata et al., 1996). As research progressed, PD1 was found to be taken part in the negative 


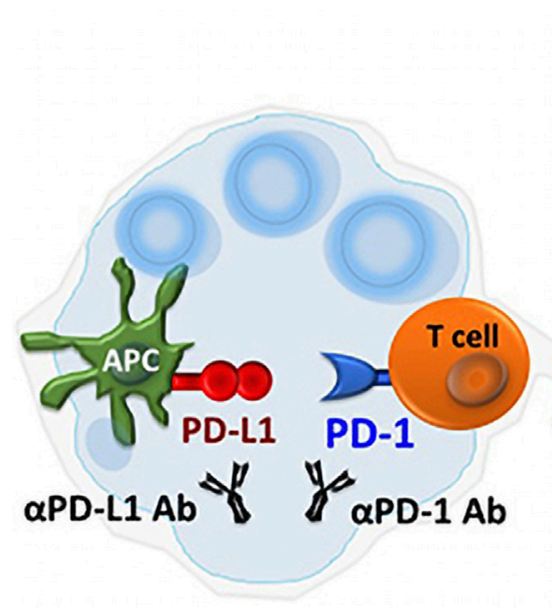

Lymph node

$1^{\text {nd }}$ Checkpoint

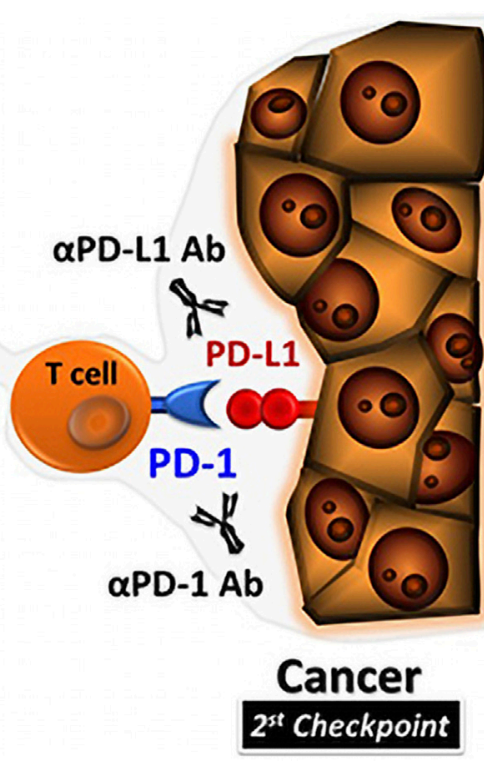

FIGURE 1 | PD1/PDL1 inhibitors in TME (Hamanishi et al., 2016).

regulation of apoptotic T cell-mediated immunological reaction through binding to PD-L1 (Nishimura et al., 1999; Greenwald et al., 2005). Studies have shown that immunotherapy is effective in treating melanoma and renal cell carcinoma, etc. (Siegel et al., 2017; Sanmamed et al., 2018; Yu et al., 2019). Recent years, checkpoint inhibitors targeting the PD1/PDL1 or Cytotoxic $\mathrm{T}$ lymphocyte associated protein 4 (CTLA-4) pathways have shown great success, and driven the development of immunotherapy (Lesokhin et al., 2015; Sharma et al., 2015; Shin and Ribas, 2015; Topalian et al., 2015; Søndergaard et al., 2018). The anti-CTLA-4 antibody ipilimumab has shown durable anti-tumor activity and prolonged survival in patients with advanced melanoma, but is prone to immune-related adverse events (IAEs) (Buchbinder et al., 2016). PD1/PDL1 inhibitors are promising immunotherapeutic agents that can achieve satisfactory efficacy for different tumor types, different treatment routes, different drug combinations and different treatment regimens (Chen et al., 2021). The incidence of PD1/PDL1 inhibitormediated IAEs was significantly lower compared to CTLA-4 blockade (Ott et al., 2013). Study shows that PD-1 pathway blockade is more efficient than CTLA-4 blockade in advanced melanoma (Farolfi et al., 2012).

\section{BIOLOGICAL FUNCTION OF PD1/PDL1 IN TUMOR IMMUNITY}

PD1 is a checkpoint protein and a composition of the CD28 family. It pertains to a group of suppressor T-cell receptors that was not expressed by $\mathrm{T}$ cells alone, but was upregulated by antigen stimulation and cytokines caused by $\mathrm{T}$ cell excitation (Kinter et al., 2008; Kulpa et al., 2013). PD1 is also expressed by
B cells, monocytes, and dendritic cells (DCs)( Keir et al., 2008), and regulates various aspects of its immune function (Thibult et al., 2013; Roy et al., 2015). PDL1 is a type 1 transmembrane glycoprotein of the B7 ligand family. Which is not only expressed on activated $\mathrm{T}$ cells and $\mathrm{B}$ cells but also on some nonhematopoietic cells (Zou et al., 2016). It is in a favorable position to regulate $\mathrm{T}$ cell function in DCs and other antigenpresenting cells (APCs). T cells recognize tumor cells in the human body and kill them, but when tumor cells recognize $\mathrm{PD} 1$ protein on $\mathrm{T}$ cells, the tumor cells will upregulate the PDL1 protein and PD1 binds to PDL1 leading to apoptosis of the T cells ( $\mathrm{Li}$ et al., 2015; Topalian et al., 2016; Tremblay et al., 2018; Li et al., 2020).

PDL1 on the surface of tumor cells can be upregulated by interferon gamma (IFN- $\gamma$ ) produced by activated T cells (Tang et al., 2018). PD1/PDL1 signal transduction pathway is a vital component of tumor immunosuppression, which can inhibit the excitation of $\mathrm{T}$ lymphocytes and strengthen the tumor cellular immune tolerance, so as to achieve tumor immune escape (Iwai et al., 2017). In summary, PD1 binds to PDL1 can diminish T cell-mediated immune surveillance, resulting in an absence of immunoreaction and even to apoptosis of $\mathrm{T}$ cells. It also inhibits tumor-infiltrating CD4+/CD8+ T cells (CD4+/ CD8+ TILs) and leads to a decrease in cytokines including tumor necrosis factor (TNF), IFN- $\gamma$ and Interleucina-2 (IL-2), so as to provide a way for cancer cells to escape the immunoreaction (Francisco et al., 2010; Daassi et al., 2020). PD1/PDL1 inhibiters unblock the immune suppression of anti-tumor $\mathrm{T}$ cells (Figure 1), which results in $\mathrm{T}$ cell multiplication and permeation into the TME and inducing an anti-tumor response (Kuzume et al., 2020). Existing antiPD1/PDL1 therapy interdicts the combination between PD1 and PDL1, and effectively activates depleted immune cells and 
triggers an anti-tumor immune response (Ribas et al., 2018; Seidel et al., 2018; Liang et al., 2021).

\section{MECHANISM OF ACTION AND TREATMENT OF PD1/PDL1 INHIBITORS Peptides/Polysaccharides and Small Molecules Target Treatment}

Recently, a great number of research has been devoted to the exploitation of peptide-based inhibitors and nonpeptidic smallmolecules targeting PD1/PDL1 (Akturk et al., 2018; Sasikumar et al., 2018). Furthermore, through structural modification of peptidomimetic inhibitors, small molecules can be developed. Compared to monoclonal antibodies, small-molecule drugs offer significant advantages (Zhang et al., 2020).

\section{Peptide-Based PD1/PDL1 Inhibitors}

The first inhibitor AUNP-12, which was reportedly patented in 2014 (Sasikumar et al., 2019), is a 29-amino acid branching peptide. In an animal study, tumor cell growth and metastasis were effectively inhibited by AUNP-12 with few adverse reactions. In addition to AUNP-12, other peptide-based PD1/PDL1 inhibitors also have been developed. For example, a small peptide mimicking a peptide containing 7-8 amino acids, showed the best bioactivity in mice infected with melanoma B16F10 cancer cells, reducing lung metastases by 64 percent. Another compound is a cyclopeptide derivative of 7-9 amino acids, characterized by the formation of a circular structure by an amide bond between the $\mathrm{N}$ and $\mathrm{C}$ ends of the amino acid residues. In Crystal Field Stabilization Energies (CFSE) detection, Sasikumar et al. found that a cyclic peptide derivative can induce the proliferation of spleen cells in mice with high expression of PDL1 in human breast MDAMB-231 cancer cells and reduce the lung metastasis of mice with melanoma B16F10 cancer cells by 54\% (Sasikumar, 2013; Sasikumar et al., 2015).

\section{Nonpeptidic PD1/PDL1 Small-Molecule Inhibitors}

The first reported small molecule inhibitor based on the PD1/ PDL1 axis was a derivative of sulfamethoxine and sulfamethimazole antibiotics (Sharpe et al., 2018), which have low cytotoxicity. They can block the PD1 signaling pathway through restraining the combination of mPD1 to mPDL2 within the micromolar concentration range. Recent years, Bristol-Myers Squibb (BMS) has revealed a large number of non-peptide small molecule inhibitors targeting PD1/PDL1 pathway (Chupak et al., 2015). Among reported compounds, the representative compounds BMS-8 had IC50 value of $146 \mathrm{nM}$, and BMS-202 had IC50 value of $18 \mathrm{nM}$. Researchers investigated the mechanism of action of BMS inhibitors and demonstrated that these inhibitors induce the dimerization of PDL1, thereby suppressing the activation of PD1 (Zak et al., 2016). Holak's team showed that some BMS compounds have structures that bind directly to PD-L1. More importantly, the combination between
PD1 and PDL1 was blocked by inducing and stabilizing the formation of PD1/PDL1 homodimer under the action of compounds (Sasikumar et al., 2016). The IC50 values of representative compounds LH1306 and LH1307 were 25 and $3.0 \mathrm{nM}$, respectively. In addition, these inhibitors can interfere with interactions between PD1/PDL1 proteins and block PD1 signal transduction in co-culture experiments (Yang and $\mathrm{Hu}$, 2019).

\section{Aptamer Therapy}

Aptamer-Drug Conjugates (APDCs) are a very promising platform. Studies have shown that APDC can deliver immunomodulators, restrict immunomodulatory costimulation to tumor regions, induce neoantigens in tumors, block depletion-induced immune checkpoints, activate functional immune cells and prolong anti-tumor immunity (Zhu and Chen, 2018). Geng et al. designed and synthesized an amphiphilic telomeric dimer, aptamer polyvalent drug conjugate (ApMDC). And described the use of ApMDC nanoparticles to enhance the antitumor reaction of $a-P D 1$ immunotherapy with targeted chemotherapy to tumors (Geng et al., 2021). They established $4 \mathrm{t} 1$ (breast cancer cell) and h22 (hepatoma carcinoma cell) tumor-bearing mouse models and draw a conclusion that the increased antitumor immunity accelerated the therapeutic reaction of $\alpha$-PD1. In one study, researchers developed a DNA inducer for PD1/PDL1 signaling pathway to reverse immune evasion and stimulate antitumor immunity (Prodeus et al., 2015). DNA aptamer blocks the interaction of $\mathrm{PD} 1 / \mathrm{PDL} 1$ by specifically binding to the extracellular domain of mouse PD1. MP7 is one of the aptamers, which can inhibit the inhibition of IL-2 secretion by primary T cells mediated by PD-L1. PEGylated MP7 directly blocks PD1 binding to PDL1. The Pegylated form of MP7 is equivalent to the antagonistic PD1 antibody, and can significantly inhibit the growth of PD-L1+ colon cancer cells in vivo for it retains the ability to block the PD1/PDL1 interaction (Ellington et al., 1990; Tuerk et al., 1990; Keefe et al., 2010).

According to another study, aptPDL1 stop the combination between PD1 and PDL1 in humans. Experiments in mouse models have shown that aptPDL1 promotes lymphocyte proliferation in vitro and inhibits tumor growth in vivo without causing significant hepatorenal toxicity. Further analysis of tumors treated with aptPD-L1 revealed increased levels of invasive $\mathrm{CD}^{+}$and $\mathrm{CD}^{+} \mathrm{T}$ cells, IL-2, TNF- $\alpha$, and IFN- $\gamma$ (Figure 2). Chemokine receptor 3(CXCR3) expression was higher in $\mathrm{CD}^{+} \mathrm{T}$ cells treated with aptPD-L1 than in tumors treated with random sequence oligonucleotide (Lai et al., 2016). Researchers have developed a novel PDL1 aptamer, a short single strand of DNA that is smaller than the PDL1 antibody, which can effectively avoid the effects of glycosylation that block PD-L1 binding. The selected adapter is more possibly to be glycosylated by PDL1 as peptide antigens, which is hopeful to provide a higher effectiveness of recognition while compared with PDL1 antibodies from extracellular cells (Huang et al., 2020). Liu's team found that in the presence of dual targets (PDL1 as a natural receptor and azide modified glycoprotein as a chemical receptor), the cyclooctyne-coupled PDL1 (D-APDL1) can be covalently 


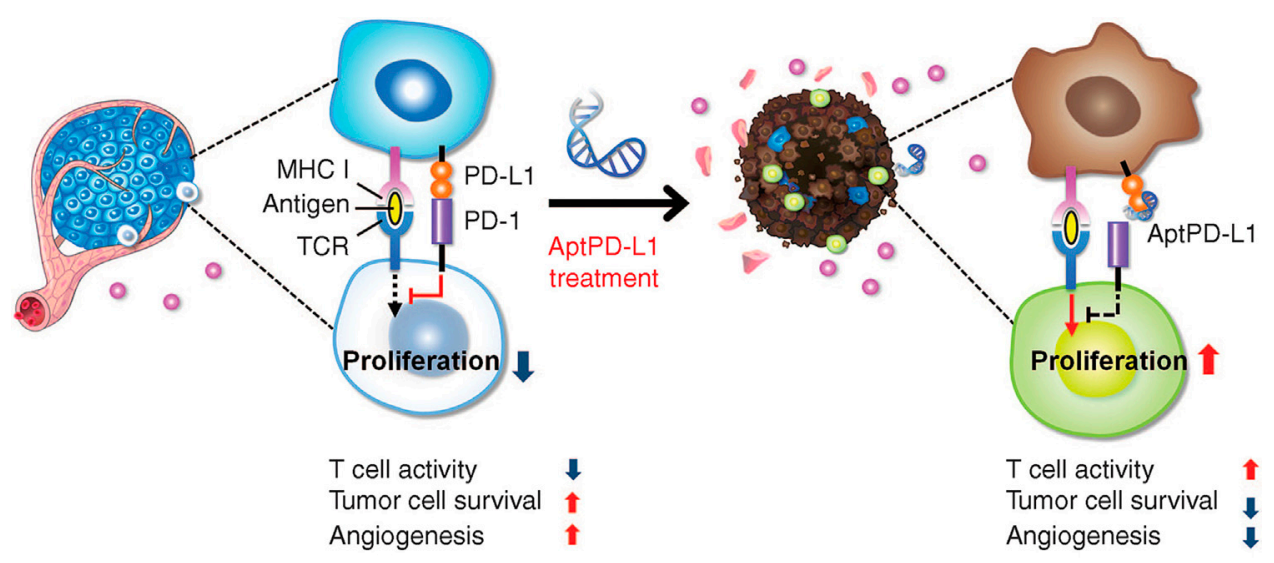

\begin{tabular}{|lll}
\hline (0) Tumor cell & Inactivated T cell & Endothelial cell \\
(0) Apoptotic tumor cell & Activated T cell & Cytokine (IL-2, TNF- $\alpha$, IFN $\gamma$, etc. $)$
\end{tabular}

FIGURE 2 | APTPD-L1 can inhibit the PD1/PDL1 interaction and weaken the inhibition of T cells (Lai et al., 2016).

coupled to the surface membrane of cancer cells through APDL1 aptamer recognition and DNA logic calculation reaction of cyclooctyne/azide biological orthogonal reaction. This in turn triggers precise and sustained $\mathrm{T}$ cell-mediated anti-tumor immunotherapy (Yang et al., 2021). Besides, they also found that this logical calculation could achieve long-term retention in the tumor by inducing covalent coupling of the PDL1 aptamer on the tumor cell surface, thus providing effective and precise checkpoint-blocking immunotherapy.

\section{Antibody Therapy}

Antibody-based inhibitors of PD1/PDL1 induce persistent tumor remission in various kinds of advanced cancer patients, making inhibition of the PD1/PDL1 signaling pathway clinically important in the treatment of tumors. So far, Food and Drug Administration (FDA) has approved six monoclonal antibodies targeting PD1 (nivolumab, pembrolizumab, and cemiplimab) or PDL1 (atezolizumab, Durvalumab and avelumab) for the treatment of hematological and solid malignancies. (Tan et al., 2016; Chen et al., 2021). Monoclonal antibodies (mAb), known as checkpoint inhibitors, overcome the shortcomings of traditional anticancer therapies and inhibit the PD1/PDL1 mutual effect. Using in vivo and in vitro studies, Lussier et al. have found that $\mathrm{T}$ cell function can be enhanced by blocking PD1 with antibodies (Lussier et al., 2015). Within tolerable limits, monoclonal antibodies can significantly reduce toxicity, reduce solid tumor size, inhibit advanced tumors and metastases, and improve overall survival in patients. Nivolumab and pembrolizumab have been given permission for the therapy of terminal melanoma, non-small cell lung cancer (NSCLC) and renal cell carcinoma (RCC) by targeting PD1 and blocking its interaction with PDL1 and PDL2 (Hughes et al., 2016; Arranz-Nicolás et al., 2021). Phase I clinical trials of pembrolizumab or atezolizumab in patients with mTNBC showed promising results, with objective response rates (ORR) of 18.5 and 33\%, respectively (Hwang et al., 2019). However, due to its long half-life and binding time with the target, it is easy to result in severe immune-related adverse reactions. Besides, $\mathrm{mAb}$ drugs are expensive, complex to produce, and difficult to store and transport. Therefore, how to use the PD1/PDL1 signaling pathway to develop simple and efficient non-monoclonal antibody treatment strategy is the focus of our current work (Pan et al., 2021).

\section{Combination Therapy}

In a study, the researchers used an immune rejection phenotype in a mouse model to discover that therapeutic application of TGF- $\beta$ blocking antibodies in combination with anti-PDL1 reduces stromal TGF- $\beta$ signaling, promotes $\mathrm{T}$ cell infiltration into tumor centers and stimulated powerful anti-tumor immunity ultimately leading to tumor extinction (Mariathasan et al., 2018). The addition of the anti-PD-L1 drug atezolizumab to NAB-paclitaxel chemotherapy has been shown to significantly improve PD-L1-positive (PD-L1+) metastases and improve overall survival (OS) in patients with advanced TNBC(Schmid et al., 2018). Combined use of CDK4/6 and PD-L1 inhibitors significantly increased the survival rate of mouse xenograft models (Zhang et al., 2015). It has been reported that combining PD1/PDL1 inhibitors with PARP inhibitors is hopeful to remarkably improve the overall efficacy of TNBC. Mechanistically, PARPI inactivates GSK3 $\beta$, thereby enhancing PARPI-mediated upregulation of PDL1 and reducing the efficacy of PARPI through cancer-associated immunosuppression. Blocking PD-L1 can restore the weakened anti-tumor immune function and enhance the antitumor effect of PARPI.

When anti-PD1/PDL1 antibody (anti-PD1/PDL1) and antiCTLA4 antibody (anti-CTLA4) are administered alone, their effectiveness is only $20-25 \%$ at most. When combined, the yield of anti-PD1/PDL1 and anti-CTLA4 could reach $60 \%$ (Wei et al., 2018). The combination of antibodies blocking PD1 and agonistic antibodies triggering the costimulatory receptor glucocorticoid induced tumor necrosis factor receptor 
(GITR) may further improve the therapeutic effect (Wang et al., 2018b). Using the mouse colon cancer cell line MC38, Wang et al. found that anti-PD-1+ anti GITR had a significantly stronger anti-tumor effect in mice than either antibody alone. The synergistic effect of anti-PD-1+ and anti-GITR depends on $\mathrm{CD}^{+} \mathrm{T}$ cells, which can directly kill cancer cells and are adept at recruiting other tumor oncogenic immune cells from tumor (Wei et al., 2018). Wang et al. used gemcitabine (GEM) and PD1/ PDL1 checkpoint inhibitor to form reactive oxygen species reactive scaffold in situ for combination treatment. They found that aPDL1-GEM scaffold induced an immunogenic tumor phenotype in mice bearing tumor, promoted immunemediated tumor regression, and prevented tumor recurrence after primary resection (Wang et al., 2018c). Gao et al. treated a mouse tumor model with an anti-PD1 antibody and an HDAC2 inhibitor. The combination of HDAC2 inhibitors and antiPD1 antibodies obviously slowed tumor growth and improved survival compared to the anti-PD1 treatment group (Gao et al., 2020).

\section{Mechanism of Drug Resistance in PD1/ PDL1 Inhibitor Therapy}

Although immune checkpoint blocking therapy has achieved great success in clinic, the response rate of immunotherapy is still low (Spranger et al., 2015; O’Donnell et al., 2017). Research has suggested that only $10-30 \%$ of the patients can produce longterm and sustained efficacy after receiving PD1/PDL1 inhibitors. The majority of patients have no obvious response to the treatment or will remain resistant to it (Andrews et al., 2019). The development of PD1/PDL1 antibody resistance involves many tumor-related processes, including PD-L1 expression, tumor neoantigen expression and delivery, related cellular signaling pathways, tumor microenvironment, and epigenetic modifications. The lack of tumor antigens causes $\mathrm{T}$ cells to fail to recognize PD1/PDL1 antibodies, leading to drug resistance. In addition, molecules that process and deliver antigens, such as MHC class I molecules and $\beta 2$ microglobulin, can also lead to resistance to immune checkpoint inhibitors (ICIs) when their genetic code is altered (D'Ursoet al., 1991; Restifo et al., 1996; Sucker et al., 2014). Aberrant cell signaling is also a factor contributing to immunotherapy resistance, such as the PI3K/ Akt pathway, Wnt/ $\beta$-linked protein pathway, JAK/STAT/IFN- $\gamma$ pathway, and mitogen-activated protein kinase (MAPK) pathway (Munn and Mellor, 2013; Lin and Zhao, 2015).

\section{Immune-Related Adverse Events}

Despite the promising efficacy of immune checkpoint inhibitors, the majority of treated patients have had immune-related adverse events (IAEs) to varying degrees (Reynolds et al., 2021). Commonly reported IAEs include rash or pruritus, gastrointestinal disorders, and endocrine disorders (Farolfi et al., 2012; Robert et al., 2015). Among these, cardiovascular toxicity is particularly severe. In recent years, reports of myocarditis associated with ICIs have increased (Moslehi et al., 2018). Myocarditis associated with ICIs often manifests as arrhythmias and can coexist with myocarditis and myasthenia gravis, with severe disease and poor prognosis (Hu et al., 2019). There is evidence that redox mechanisms are the main mechanism responsible for cardiotoxicity (Tocchetti et al., 2019). It has been shown that ICI treatment group had a higher incidence of cardiovascular adverse reactions than the non-ICI treatment group, and the incidence of cardiovascular adverse reactions was higher in patients treated with the combination of ICI + ICI than with ICI monotherapy. CTLA4 is prone to immune-related adverse reactions such as rash, diarrhea, colitis, hepatotoxicity and endocrine disorders (Hodi et al., 2010; Gangadhar et al., 2014), as well as cardiotoxicity including pericarditis and myocarditis (Geisler et al., 2015; Heinzerling et al., 2016). As for PD1/PDL1 inhibitors, myocarditis has been reported after treatment with nivolumab (Honton et al., 2014) or pembrolizumab (Läubli et al., 2015). Wang et al. found significantly higher rates of colitis and diarrhea after receiving the combination of ipilimumab and PD1/PDL1 inhibitors than with a single agent (Wang et al., 2017).

\section{CONCLUSION AND PROSPECT}

Over the past 20 years since the discovery of PD1, numerous experimental studies have proved the clinical efficacy of PD1 blockers in a wide range of solid and hematologic malignancies, offering promising prospects for cancer patients (Errico, 2015). In addition, reports based on the clinical application of PD1 inhibitors have elucidated the mechanism of tumor immune escape and confirmed the general significance of tumor immune monitoring and tumor immune editing (Burnet, 1967; Schreiber et al., 2011). Nevertheless, there is still a need for a large number of basic and exploratory studies on the prediction of tumor biomarkers, as well as the efficacy of drug therapy and adverse drug reactions. However, this does not prevent PD1/PDL1 from being a key area of research. For the reason that PD1/PDL1 plays a crucial role in most cancers, the development of immunotherapy with blocking agents will undoubtedly be a huge opportunity and challenge. Due to the occurrence of drug resistance, the efficacy of immunosuppressive therapy is poor. We hope that future studies can minimize drug resistance, reduce the occurrence of immune-related adverse events and improve the efficacy of immunotherapy. We believe that as research progresses, personalized immunotherapy will be further developed in the clinic to bring hope to cancer patients.

\section{AUTHOR CONTRIBUTIONS}

JL summarized the literature and drafted the manuscript. YL and $\mathrm{WZ}$ provided revisions. JW, ZC, and ZZ conceived of the review and provided feedback. All of them approve the final version and participate in the submission.

\section{FUNDING}

This work was supported by National Nature Science Foundation of China (No. 21775061), Shandong Provincial Key Research and 
Development Program (2019GSF108253), Open Funds of State Key Laboratory of ChemoBiosensing and Chemometrics

\section{REFERENCES}

Agata, Y., Kawasaki, A., Nishimura, H., Ishida, Y., Tsubata, T., Yagita, H., et al. (1996). Expression of the PD-1 Antigen on the Surface of Stimulated Mouse T and B Lymphocytes. Int. Immunol. 8, 765-772. doi:10.1093/intimm/8.5.765

Akturk, H. K., and Michels, A. W. (2018). Adverse Events Associated with Immune Checkpoint Blockade. N. Engl. J. Med. 378 (12), 1163-1164. doi:10.1056/ NEJMc1801663Andrews

Arranz-Nicolás, J., Martin-Salgado, M., Adán-Barrientos, I., Liébana, R., del Carmen Moreno-Ortíz, M., Leitner, J., et al. (2021). Diacylglycerol Kinase a Inhibition Cooperates with PD-1-Targeted Therapies to Restore the T Cell Activation Program. Cancer Immunol. Immunother.. doi:10.1007/s00262-021-02924-5

Buchbinder, E. I., and Desai, A. (2016). CTLA-4 and PD-1 Pathways: Similarities, Differences, and Implications of Their Inhibition. Am. J. Clin. Oncol. 39 (1), 98-106. doi:10.1097/COC.0000000000000239

Burnet, F. M. (1967). Immunological Aspects of Malignant Disease. Lancet 1 (7501), 1171-1174. doi:10.1016/s0140-6736(67)92837-1

Carlomagno, N., Incollingo, P., Tammaro, V., Peluso, G., Rupealta, N., Chiacchio, G., et al. (2017). Diagnostic, Predictive, Prognostic, and Therapeutic Molecular Biomarkers in Third Millennium: A Breakthrough in Gastric Cancer. Biomed. Res. Int. 2017, 7869802. doi:10.1155/2017/7869802

Chen, S., Zhang, Z., Zheng, X., Tao, H., Zhang, S., Ma, J., et al. (2021). Response Efficacy of PD-1 and PD-L1 Inhibitors in Clinical Trials: A Systematic Review and Meta-Analysis. Front. Oncol. 11, 562315. doi:10.3389/fonc.2021.562315

Chupak, L. S., and Zheng, X. (2015). Compounds Useful as Immunomodulators. WO Patent: WO2015034820.

D’Urso, C. M., Wang, Z. G., Cao, Y., Tatake, R., Zeff, R. A., and Ferrone, S. (1991). Lack of HLA Class I Antigen Expression by Cultured Melanoma Cells FO-1 Due to a Defect in B2m Gene Expression. J. Clin. Invest. 87 (1), 284-292. doi:10.1172/JCI114984

Daassi, D., Mahoney, K. M., and Freeman, G. J. (2020). The Importance of Exosomal PDL1 in Tumour Immune Evasion. Nat. Rev. Immunol. 20 (4), 209-215. doi:10.1038/s41577-019-0264-y

Dong, H., Strome, S. E., Salomao, D. R., Tamura, H., Hirano, F., Flies, D. B., et al. (2002). Tumor-associated B7-H1 Promotes T-Cell Apoptosis: a Potential Mechanism of Immune Evasion. Nat. Med. 8, 793-800. doi:10.1038/nm730

Dunn, G. P., Bruce, A. T., Ikeda, H., Old, L. J., and Schreiber, R. D. (2002). Cancer Immunoediting: from Immunosurveillance to Tumor Escape. Nat. Immunol. 3 (11), 991-998. doi:10.1038/ni1102-991

Ellington, A. D., and Szostak, J. W. (1990). In Vitro selection of RNA Molecules that Bind Specific Ligands. Nature 346 (6287), 818-822. doi:10.1038/346818a0

Errico, A. (2015). Immunotherapy: PD-1-PD-L1 axis: Efficient Checkpoint Blockade against Cancer. Nat. Rev. Clin. Oncolclinical Oncology 12 (2), 63. doi:10.1038/nrclinonc.2014.221

Farolfi, A., Ridolfi, L., Guidoboni, M., Nicoletti, S. V., Piciucchi, S., Valmorri, L., et al. (2012). Ipilimumab in Advanced Melanoma: Reports of Long-Lasting Responses. Melanoma Res. 22 (3), 263-270. doi:10.1097/ CMR.0b013e328353e65c

Francisco, L. M., Sage, P. T., and Sharpe, A. H. (2010). The PD-1 Pathway in Tolerance and Autoimmunity. Immunol. Rev. 236, 219-242. doi:10.1111/ j.1600-065X.2010.00923.x

Gangadhar, T. C., and Vonderheide, R. H. (2014). Mitigating the Toxic Effects of Anticancer Immunotherapy. Nat. Rev. Clin. Oncol. 11 (2), 91-99. doi:10.1038/ nrclinonc.2013.245

Gao, Y., Nihira, N. T., Bu, X., Chu, C., Zhang, J., Kolodziejczyk, A., et al. (2020). Acetylation-dependent Regulation of PD-L1 Nuclear Translocation Dictates the Efficacy of Anti-PD-1 Immunotherapy. Nat. Cel Biol 22 (9), 1064-1075. doi:10.1038/s41556-020-0562-4

Geisler, B. P., Raad, R. A., Esaian, D., Sharon, E., and Schwartz, D. R. (2015). Apical Ballooning and Cardiomyopathy in a Melanoma Patient Treated with Ipilimumab: a Case of Takotsubo-like Syndrome. J. Immunother. Cancer 3, 4. doi:10.1186/s40425-015-0048-2

Geng, Z., Wang, L., Liu, K., Liu, J., and Tan, W. (2021). Enhancing Anti-PD-1 Immunotherapy by Nanomicelles Self-Assembled from Multivalent Aptamer
(2019016), Science Foundation of Innovative Research Teams of Accurate Disease Identification and Targeted Therapy (22202105).

Drug Conjugates. Angew. Chem. Int. Ed. Engl. 60 (28), 15459-15465. doi:10.1002/anie.202102631

Greenwald, R. J., Freeman, G. J., and Sharpe, A. H. (2005). The B7 Family Revisited. Annu. Rev. Immunol. 23, 515-548. doi:10.1146/ annurev.immunol.23.021704.115611

Hamanishi, J., Mandai, M., Matsumura, N., Abiko, K., Baba, T., and Konishi, I. (2016). PD-1/PD-L1 Blockade in Cancer Treatment: Perspectives and Issues. Int. J. Clin. Oncol. 21 (3), 462-473. doi:10.1007/s10147-016-0959-z

Heinzerling, L., Ott, P. A., Hodi, F. S., Husain, A. N., Tajmir-Riahi, A., Tawbi, H., et al. (2016). Cardiotoxicity Associated with CTLA4 and PD1 Blocking Immunotherapy. J. Immunother. Cancer 4, 50. doi:10.1186/s40425-016-0152-y

Hodi, F. S., O'Day, S. J., McDermott, D. F., Weber, R. W., Sosman, J. A., Haanen, J. B., et al. (2010). Improved Survival with Ipilimumab in Patients with Metastatic Melanoma. N. Engl. J. Med. 363 (8), 711-723. doi:10.1056/ NEJMoa1003466

Honton, B., Despas, F., Dumonteil, N., Rouvellat, C., Roussel, M., Carrie, D., et al. (2014). Bortezomib and Heart Failure: Case-Report and Review of the French Pharmacovigilance Database. Fundam. Clin. Pharmacol. 28 (3), 349-352. doi: $10.1111 /$ fcp. 12039

Hu, J. R., Florido, R., Lipson, E. J., Naidoo, J., Ardehali, R., Tocchetti, C. G., et al. (2019). Cardiovascular Toxicities Associated with Immune Checkpoint Inhibitors. Cardiovasc. Res. 115 (5), 854-868. doi:10.1093/cvr/cvz026

Huang, M., Yang, J., Wang, T., Song, J., Xia, J., Wu, L., et al. (2020). Homogeneous, Low-Volume, Efficient, and Sensitive Quantitation of Circulating Exosomal PD-L1 for Cancer Diagnosis and Immunotherapy Response Prediction. Angew. Chem. Int. Ed. Engl. 59 (12), 4800-4805. doi:10.1002/anie.201916039

Hughes, P. E., Caenepeel, S., and Wu, L. C. (2016). Targeted Therapy and Checkpoint Immunotherapy Combinations for the Treatment of Cancer. Trends Immunol. 37 (7), 462-476. doi:10.1016/j.it.2016.04.010

Hwang, S. Y., Park, S., and Kwon, Y. (2019). Recent Therapeutic Trends and Promising Targets in Triple Negative Breast Cancer. Pharmacol. Ther. 199, 30-57. doi:10.1016/j.pharmthera.2019.02.006

Inaguma, S., Lasota, J., Wang, Z., Czapiewski, P., Langfort, R., Rys, J., et al. (2018). Expression of ALCAM (CD166) and PD-L1 (CD274) Independently Predicts Shorter Survival in Malignant Pleural Mesothelioma. Hum. Pathol. 71, 1-7. doi:10.1016/j.humpath.2017.04.032

Ishida, Y., Agata, Y., Shibahara, K., and Honjo, T. (1992). Induced Expression of PD-1, a Novel Member of the Immunoglobulin Gene Superfamily, upon Programmed Cell Death. EMBO J. 11 (11), 3887-3895. doi:10.1002/j.14602075.1992.tb05481.x

Iwai, Y., Hamanishi, J., Chamoto, K., and Honjo, T. (2017). Cancer Immunotherapies Targeting the PD-1 Signaling Pathway. J. Biomed. Sci. 24 (1), 26. doi:10.1186/s12929-017-0329-9

Iwai, Y., Ishida, M., Tanaka, Y., Okazaki, T., Honjo, T., and Minato, N. (2002). Involvement of PD-L1 on Tumor Cells in the Escape from Host Immune System and Tumor Immunotherapy by PD-L1 Blockade. Proc. Natl. Acad. Sci. U S A. 99, 12293-12297. doi:10.1073/pnas.192461099

Keefe, A. D., Pai, S., and Ellington, A. (2010). Aptamers as Therapeutics. Nat. Rev. Drug Discov. 9 (7), 537-550. doi:10.1038/nrd3141

Keir, M. E., Butte, M. J., Freeman, G. J., and Sharpe, A. H. (2008). PD-1 and its Ligands in Tolerance and Immunity. Annu. Rev. Immunol. 26, 677-704. doi:10.1146/annurev.immunol.26.021607.090331

Kinter, A. L., Godbout, E. J., McNally, J. P., Sereti, I., Roby, G. A., O'Shea, M. A., et al. (2008). The Common Gamma-Chain Cytokines IL-2, IL-7, IL-15, and IL-21 Induce the Expression of Programmed Death-1 and its Ligands. J. Immunol. 181 (10), 6738-6746. doi:10.4049/ jimmunol.181.10.6738

Kulpa, D. A., Lawani, M., Cooper, A., Peretz, Y., Ahlers, J., and Sékaly, R. P. (2013). PD-1 Coinhibitory Signals: the Link between Pathogenesis and protection. Semin. Immunol. 25 (3), 219-227. doi:10.1016/j.smim.2013.02.002

Kuzume, A., Chi, S., Yamauchi, N., and Minami, Y. (2020). Immune-Checkpoint Blockade Therapy in Lymphoma. Int. J. Mol. Sci. 21 (15), 5456. doi:10.3390/ ijms21155456 
Lai, W. Y., Huang, B. T., Wang, J. W., Lin, P. Y., and Yang, P. C. (2016). A Novel PD-L1-Targeting Antagonistic DNA Aptamer with Antitumor Effects. Mol. Ther. Nucleic Acids 5 (12), e397. doi:10.1038/mtna.2016.102

Läubli, H., Balmelli, C., Bossard, M., Pfister, O., Glatz, K., and Zippelius, A. (2015). Acute Heart Failure Due to Autoimmune Myocarditis under Pembrolizumab Treatment for Metastatic Melanoma. J. Immunother. Cancer 3, 11. doi:10.1186/s40425-015-0057-1

Lawrence, P., Yano, H., and Vignali, D. A. A. (2019). Inhibitory Receptors and Ligands beyond PD-1, PD-L1 and CTLA-4: Breakthroughs or Backups. Nat. Immunol. 20 (11), 1425-1434. doi:10.1038/s41590-019-0512-0

Lesokhin, A. M., Callahan, M. K., Postow, M. A., and Wolchok, J. D. (2015). On Being Less Tolerant: Enhanced Cancer Immunosurveillance Enabled by Targeting Checkpoints and Agonists of T Cell Activation. Sci. Transl Med. 7, 280sr1. doi:10.1126/scitranslmed.3010274

Li, X. S., Li, J. W., Li, H., and Jiang, T. (2020). Prognostic Value of Programmed Cell Death Ligand 1 (PD-L1) for Hepatocellular Carcinoma: a Meta-Analysis. Biosci. Rep. 40 (4), BSR20200459. doi:10.1042/BSR20200459

Li, Y., Fang, M., Zhang, J., Wang, J., Song, Y., Shi, J., et al. (2015). Hydrogel Dual Delivered Celecoxib and Anti-PD-1 Synergistically Improve Antitumor Immunity. Oncoimmunology 5 (2), e1074374. doi:10.1080/ 2162402X.2015.1074374

Liang, B., Hu, X., Ding, Y., and Liu, M. (2021). Tumor-derived Exosomes in the PD-1/pd-L1 axis: Significant Regulators as Well as Promising Clinical Targets. J. Cel Physiol 236 (6), 4138-4151. doi:10.1002/jcp.30197

Lin, R. L., and Zhao, L. J. (2015). Mechanistic Basis and Clinical Relevance of the Role of Transforming Growth Factor- $\beta$ in Cancer. Cancer Biol. Med. 12 (4), 385-393. doi:10.7497/j.issn.2095-3941.2015.0015

Lussier, D. M., O'Neill, L., Nieves, L. M., McAfee, M. S., Holechek, S. A., Collins, A. W., et al. (2015). Enhanced T-Cell Immunity to Osteosarcoma through Antibody Blockade of PD-1/pd-L1 Interactions. J. Immunother. 38 (3), 96-106. doi:10.1097/CJI.0000000000000065

Mariathasan, S., Turley, S. J., Nickles, D., Castiglioni, A., Yuen, K., Wang, Y., et al. (2018). TGF $\beta$ Attenuates Tumour Response to PD-L1 Blockade by Contributing to Exclusion of $\mathrm{T}$ Cells. Nature 554 (7693), 544-548. doi:10.1038/nature25501

Moslehi, J. J., Salem, J. E., Sosman, J. A., Lebrun-Vignes, B., and Johnson, D. B. (2018). Increased Reporting of Fatal Immune Checkpoint Inhibitor-Associated Myocarditis. Lancet 391 (10124), 933. doi:10.1016/S0140-6736(18)30533-6

Munn, D. H., and Mellor, A. L. (2013). Indoleamine 2,3 Dioxygenase and Metabolic Control of Immune Responses. Trends Immunol. 34 (3), 137-143. doi:10.1016/j.it.2012.10.001

Nishimura, H., Nose, M., Hiai, H., Minato, N., and Honjo, T. (1999). Development of Lupus-like Autoimmune Diseases by Disruption of the PD-1 Gene Encoding an ITIM Motif-Carrying Immunoreceptor. Immunity 11, 141-151. doi:10.1016/s1074-7613(00)80089-8

O’Donnell, J. S., Long, G. V., Scolyer, R. A., Teng, M. W., and Smyth, M. J. (2017). Resistance to PD1/PDL1 Checkpoint Inhibition. Cancer Treat. Rev. 52, 71-81. doi:10.1016/j.ctrv.2016.11.007

Ott, P. A., Hodi, F. S., and Robert, C. (2013). CTLA-4 and PD-1/pd-L1 Blockade: New Immunotherapeutic Modalities with Durable Clinical Benefit in Melanoma Patients. Clin. Cancer Res. 19 (19), 5300-5309. doi:10.1158/10780432.CCR-13-0143

Pan, C., Yang, H., Lu, Y., Hu, S., Wu, Y., He, Q., et al. (2021). Recent advance of Peptide-Based Molecules and Nonpeptidic Small-Molecules Modulating PD-1/ pd-L1 Protein-Protein Interaction or Targeting PD-L1 Protein Degradation. Eur. J. Med. Chem. 213, 113170. doi:10.1016/j.ejmech.2021.113170

Prestipino, A., and Zeiser, R. (2019). Clinical Implications of Tumor-Intrinsic Mechanisms Regulating PD-L1. Sci. Transl Med. 11 (478), eaav4810. doi:10.1126/scitranslmed.aav4810

Prodeus, A., Abdul-Wahid, A., Fischer, N. W., Huang, E. H., Cydzik, M., and Gariépy, J. (2015). Targeting the PD-1/pd-L1 Immune Evasion Axis with DNA Aptamers as a Novel Therapeutic Strategy for the Treatment of Disseminated Cancers. Mol. Ther. Nucleic Acids 4 (4), e237. doi:10.1038/mtna.2015.11

Restifo, N. P., Marincola, F. M., Kawakami, Y., Taubenberger, J., Yannelli, J. R., and Rosenberg, S. A. (1996). Loss of Functional Beta 2-microglobulin in Metastatic Melanomas from Five Patients Receiving Immunotherapy. J. Natl. Cancer Inst. 88 (2), 100-108. doi:10.1093/jnci/88.2.100

Reynolds, K. L., Arora, S., Elayavilli, R. K., Louv, W. C., Schaller, T. H., Khandelwal, A., et al. (2021). Immune-related Adverse Events Associated with Immune
Checkpoint Inhibitors: a Call to Action for Collecting and Sharing Clinical Trial and Real-World Data. J. Immunother. Cancer 9 (7), e002896. doi:10.1136/jitc2021-002896

Ribas, A., and Wolchok, J. D. (2018). Cancer Immunotherapy Using Checkpoint Blockade. Science 359 (6382), 1350-1355. doi:10.1126/science.aar4060

Robert, C., Schachter, J., Long, G. V., Arance, A., Grob, J. J., Mortier, L., et al. (2015). Pembrolizumab versus Ipilimumab in Advanced Melanoma. N. Engl. J. Med. 372 (26), 2521-2532. doi:10.1056/NEJMoa1503093

Roy, S., Gupta, P., Palit, S., Basu, M., Ukil, A., and Das, P. K. (2015). The Role of PD-1 in Regulation of Macrophage Apoptosis and its Subversion by Leishmania Donovani. Clin. Transl Immunol. 6 (5), e137. doi:10.1038/cti.2017.12

Sanmamed, M. F., and Chen, L. (2018). A Paradigm Shift in Cancer Immunotherapy: From Enhancement to Normalization. Cell 175, 313-326. doi:10.1016/j.cell.2018.09.03521

Sasikumar, P. G., and Ramachandra, M. (2018). Small-molecule Immune Checkpoint Inhibitors Targeting PD-1/pd-L1 and Other Emerging Checkpoint Pathways. BioDrugs 32 (5), 481-497. doi:10.1007/s40259-0180303-4

Sasikumar, P. G., Ramachandra, R. K., Adurthi, S., Dhudashiya, A. A., Vadlamani, S., Vemula, K., et al. (2019). A Rationally Designed Peptide Antagonist of the PD-1 Signaling Pathway as an Immunomodulatory Agent for Cancer Therapy. Mol. Cancer Ther. 18 (6), 1081-1091. doi:10.1158/1535-7163.MCT-18-0737

Sasikumar, P. G., and Ramachandra, M. (2013). Immunomodulating Cyclic Compounds from the BC Loop of Human PD1. WO 2013144704.

Sasikumar, P. G., Ramachandra, M., and Naremaddepalli, S. S. (2016). 1,3,4Oxadiazole and Thiadiazole Compounds as Immunomodulators. WO2016142852.

Sasikumar, P. G., and Ramachandra, M. (2015). Therapeutic Immunomodulating Compounds. WO2015044900.

Schmid, P., Adams, S., Rugo, H. S., Schneeweiss, A., Barrios, C. H., Iwata, H., et al. (2018). Atezolizumab and Nab-Paclitaxel in Advanced Triple-Negative Breast Cancer. N. Engl. J. Med. 379 (22), 2108-2121. doi:10.1056/NEJMoa1809615

Schreiber, R. D., Old, L. J., and Smyth, M. J. (2011). Cancer Immunoediting: Integrating Immunity's Roles in Cancer Suppression and Promotion. Science 331, 1565-1570. doi:10.1126/science.1203486

Seidel, J. A., Otsuka, A., and Kabashima, K. (2018). Anti-PD-1 and Anti-CTLA-4 Therapies in Cancer: Mechanisms of Action, Efficacy, and Limitations. Front. Oncol. 8, 86. doi:10.3389/fonc.2018.00086

Sharma, P., and Allison, J. P. (2015). The Future of Immune Checkpoint Therapy. Science 348 (6230), 56-61. doi:10.1126/science.aaa8172

Sharpe, A. H., and Pauken, K. E. (2018). The Diverse Functions of the PD1 Inhibitory Pathway. Nat. Rev. Immunol. 18 (3), 153-167. doi:10.1038/nri.2017.108

Shin, D. S., and Ribas, A. (2015). The Evolution of Checkpoint Blockade as a Cancer Therapy: What's Here, What's Next?. Curr. Opin. Immunol. 33, 23-35. doi:10.1016/j.coi.2015.01.006

Siegel, R. L., Miller, K. D., and Jemal, A. (2017). Cancer Statistics, 2017. CA Cancer J. Clin. 67, 7-30. doi:10.3322/caac.21387

Søndergaard, J. N., van Heeringen, S. J., Looman, M. W. G., Tang, C., Triantis, V., Louche, P., et al. (2018). Dendritic Cells Actively Limit Interleukin-10 Production under Inflammatory Conditions via DC-SCRIPT and Dual-Specificity Phosphatase 4. Front. Immunol. 9, 1420. doi:10.3389/fimmu.2018.01420

Spranger, S., Bao, R., and Gajewski, T. F. (2015). Melanoma-intrinsic $\beta$-catenin Signalling Prevents Anti-tumour Immunity. Nature 523, 231-235. doi:10.1038/ nature14404

Sucker, A., Zhao, F., Real, B., Heeke, C., Bielefeld, N., Maßen, S., et al. (2014). Genetic Evolution of T-Cell Resistance in the Course of Melanoma Progression. Clin. Cancer Res. 20 (24), 6593-6604. doi:10.1158/10780432.CCR-14-0567

Takeuchi, H., Konnai, S., Maekawa, N., Minato, E., Ichikawa, Y., Kobayashi, A., et al. (2020). Expression Analysis of Canine CMTM6 and CMTM4 as Potential Regulators of the PD-L1 Protein in Canine Cancers. Front. Vet. Sci. 7, 330. doi: $10.3389 /$ fvets.2020.00330

Tan, S., Zhang, C. W., and Gao, G. F. (2016). Seeing Is Believing: Anti-PD-1/pd-L1 Monoclonal Antibodies in Action for Checkpoint Blockade Tumor Immunotherapy. Signal. Transduct Target. Ther. 1, 16029. doi:10.1038/ sigtrans.2016.29

Tang, H., Liang, Y., Anders, R. A., Taube, J. M., Qiu, X., Mulgaonkar, A., et al. (2018). PD-L1 on Host Cells Is Essential for PD-L1 Blockade- 
Mediated Tumor Regression. J. Clin. Invest. 128 (2), 580-588. doi:10.1172/JCI96061

Thibult, M. L., Mamessier, E., Gertner-Dardenne, J., Pastor, S., Just-Landi, S., Xerri, L., et al. (2013). PD-1 Is a Novel Regulator of Human B-Cell Activation. Int. Immunol. 25 (2), 129-137. doi:10.1093/intimm/dxs098

Tocchetti, C. G., Cadeddu, C., Di Lisi, D., Femminò, S., Madonna, R., Mele, D., et al. (2019). From Molecular Mechanisms to Clinical Management of Antineoplastic Drug-Induced Cardiovascular Toxicity: A Translational Overview. Antioxid. Redox Signal. 30 (18), 2110-2153. doi:10.1089/ars.2016.6930

Topalian, S. L., Drake, C. G., and Pardoll, D. M. (2015). Immune Checkpoint Blockade: a Common Denominator Approach to Cancer Therapy. Cancer Cell 27 (4), 450-461. doi:10.1016/j.ccell.2015.03.001

Topalian, S. L., Taube, J. M., Anders, R. A., and Pardoll, D. M. (2016). Mechanismdriven Biomarkers to Guide Immune Checkpoint Blockade in Cancer Therapy. Nat. Rev. Cancer 16 (5), 275-287. doi:10.1038/nrc.2016.36

Tremblay-LeMay, R., Rastgoo, N., and Chang, H. (2018). Modulating PD-L1 Expression in Multiple Myeloma: an Alternative Strategy to Target the PD1/pd-L1 Pathway. J. Hematol. Oncol. 11 (1), 46. doi:10.1186/s13045-0180589-1

Tuerk, C., and Gold, L. (1990). Systematic Evolution of Ligands by Exponential Enrichment: RNA Ligands to Bacteriophage T4 DNA Polymerase. Science 249, 505-510. doi:10.1126/science.2200121

Wang, B., Zhang, W., Jankovic, V., Golubov, J., Poon, P., Oswald, E. M., et al. (2018a). Combination Cancer Immunotherapy Targeting PD-1 and GITR Can rescue CD8+ T Cell Dysfunction and Maintain Memory Phenotype. Sci. Immunol. 3 (29), eaat7061. doi:10.1126/sciimmunol.aat7061

Wang, C., Kar, S., Lai, X., Cai, W., Arfuso, F., Sethi, G., et al. (2018b). Triple Negative Breast Cancer in Asia: An Insider's View. Cancer Treat. Rev. 62, 29-38. doi:10.1016/j.ctrv.2017.10.014

Wang, C., Wang, J., Zhang, X., Yu, S., Wen, D., Hu, Q., et al. (2018c). In Situ formed Reactive Oxygen Species-Responsive Scaffold with Gemcitabine and Checkpoint Inhibitor for Combination Therapy. Sci. Transl Med. 10 (429), eaan3682. doi:10.1126/scitranslmed.aan3682

Wang, D. Y., Ye, F., Zhao, S., and Johnson, D. B. (2017). Incidence of Immune Checkpoint Inhibitor-Related Colitis in Solid Tumor Patients: A Systematic Review and Meta-Analysis. Oncoimmunology 6 (10), e1344805. doi:10.1080/ 2162402X.2017.1344805

Wei, S. C., Duffy, C. R., and Allison, J. P. (2018). Fundamental Mechanisms of Immune Checkpoint Blockade Therapy. Cancer Discov. 8 (9), 1069-1086. doi:10.1158/2159-8290.CD-18-0367

Yang, J., and Hu, L. (2019). Immunomodulators Targeting the PD-1/pdL1 Protein-Protein Interaction: from Antibodies to Small Molecules. Med. Res. Rev. 39 (1), 265-301. doi:10.1002/med.21530
Yang, Y., Xu, J., Sun, Y., Mo, L., Liu, B., Pan, X., et al. (2021). Aptamer-Based Logic Computing Reaction on Living Cells to Enable Non-antibody Immune Checkpoint Blockade Therapy. J. Am. Chem. Soc. 143 (22), 8391-8401. doi:10.1021/jacs.1c02016

Yu, X., Huang, X., Chen, X., Liu, J., Wu, C., Pu, Q., et al. (2019). Characterization of a Novel Anti-human Lymphocyte Activation Gene 3 (LAG-3) Antibody for Cancer Immunotherapy. MAbs 11 (6), 1139-1148. doi:10.1080/19420862.2019.1629239

Zak, K. M., Grudnik, P., Guzik, K., Zieba, B. J., Musielak, B., Dömling, A., et al. (2016). Structural Basis for Small Molecule Targeting of the Programmed Death Ligand 1 (PD-L1). Oncotarget 7 (21), 30323-30335. doi:10.18632/ oncotarget.87310.18632/oncotarget.8730

Zhang, J., Bu, X., Wang, H., Zhu, Y., Geng, Y., Nihira, N. T., et al. (2018). Cyclin D-CDK4 Kinase Destabilizes PD-L1 via Cullin 3-SPOP to Control Cancer Immune Surveillance. Nature 553, 91-95. doi:10.1038/nature25015

Zhang, J. Y., Yan, Y. Y., Li, J. J., Adhikari, R., and Fu, L. W. (2020). PD-1/PD-L1 Based Combinational Cancer Therapy: Icing on the Cake. Front. Pharmacol. 11, 722. doi:10.3389/fphar.2020.00722

Zhang, S., Bai, X., and Shan, F. (2020). The Progress and Confusion of Anti-pd1/ pd-L1 Immunotherapy for Patients with Advanced Non-small Cell Lung Cancer. Int. Immunopharmacol 80, 106247. doi:10.1016/j.intimp.2020.106247

Zhu, G., and Chen, X. (2018). Aptamer-based Targeted Therapy. Adv. Drug Deliv. Rev. 134, 65-78. doi:10.1016/j.addr.2018.08.005

Zou, W., Wolchok, J. D., and Chen, L. (2016). PD-L1 (B7-H1) and PD-1 Pathway Blockade for Cancer Therapy: Mechanisms, Response Biomarkers, and Combinations. Sci. Transl Med. 8, 328rv4. doi:10.1126/scitranslmed.aad7118

Conflict of Interest: The authors declare that the research was conducted in the absence of any commercial or financial relationships that could be construed as a potential conflict of interest.

Publisher's Note: All claims expressed in this article are solely those of the authors and do not necessarily represent those of their affiliated organizations, or those of the publisher, the editors and the reviewers. Any product that may be evaluated in this article, or claim that may be made by its manufacturer, is not guaranteed or endorsed by the publisher.

Copyright $\odot 2021 \mathrm{Liu}$, Chen, Li, Zhao, Wu and Zhang. This is an open-access article distributed under the terms of the Creative Commons Attribution License (CC BY). The use, distribution or reproduction in other forums is permitted, provided the original author(s) and the copyright owner(s) are credited and that the original publication in this journal is cited, in accordance with accepted academic practice. No use, distribution or reproduction is permitted which does not comply with these terms. 